\title{
INTEGRALIDADE DO CUIDADO NA FORMAÇÃO DO ENFERMEIRO: VISÕES E VIVÊNCIAS DO ACADÊMICO DE ENFERMAGEM
}

Ana Thais Santana Santos ${ }^{1}$, Caroline Bispo de Oliveira ${ }^{1}$, Meiriane do Carmo Passos ${ }^{2}$, Aglae da Silva Araujo Andrade ${ }^{1}$, Fernanda Costa Martins Gallotti ${ }^{3}$

Objetivo: Conhecer as concepções dos acadêmicos de enfermagem sobre integralidade no cuidado a partir da vivência ao longo do curso e identificar como ocorre a aplicação desse principio na formação do enfermeiro. Metodologia: pesquisa qualitativa, descritiva e exploratória, realizada com 09 acadêmicos de enfermagem de uma universidade do nordeste do Brasil, em junho de 2017, a partir de entrevistas semiestruturadas, submetidas a análise dos dados mediante a análise proposta por Minayo. Resultados: emergiram duas categorias: Integralidade no cuidado em saúde; Formação do enfermeiro na perspectiva da integralidade. Conclusão: $O$ cuidado integral é uma construção gradual e ocorre a partir de relações e reformulação de modelos unidimensionais. Para tal, é necessário associar o conhecimento e a pratica para disseminação e aplicabilidade, imprescindivel à abordagem desde a formação acadêmica.

Descritores: Integralidade em saúde; Cuidados de enfermagem; Ensino.

\section{INTEGRALITY OF CARE IN THE TRAINING OF NURSES: VISIONS AND EXPERIENCES OF NURSING STUDENTS}

Objective: To know the conceptions of nursing academics about integrality in care based on the experience during the course and to identify how it occurs to the application of this principle in the training of nurses. Methodology: qualitative, descriptive and exploratory research, carried out with 09 nursing academics from a university in the northeast of Brazil, in June 2017, from semi-structured interviews, submitted to analysis of the data through the analysis proposed by Minayo. Results: two categories emerged: Integrality in health care; Nursing training in the perspective of integrality. Conclusion: Integral care is a gradual construction and is based on relationships and reformulation of one-dimensional models. To do this, it is necessary to associate knowledge and practice for dissemination and applicability, which is essential for the approach from academic training.

Descriptors: Integrality in health; Nursing care; Teaching.

\section{INTEGRALIDAD DEL CUIDADO EN LA FORMACIÓN DEL ENFERMERO: VISIONES Y VIVENCIAS DEL ACADÉMICO DE ENFERMERÍA}

Objetivo: Conocer las concepciones de los académicos de enfermería sobre integralidad en el cuidado a partir de la vivencia a lo largo del curso e identificar cómo ocurre a la aplicación de ese principio en la formación del enfermero. Metodología: investigación cualitativa, descriptiva y exploratoria, realizada con 09 académicos de enfermeria de una universidad del nordeste de Brasil, en junio de 2017, a partir de entrevistas semiestructuradas, sometidas al análisis de los datos mediante el análisis propuesto por Minayo. Resultados: emergieron dos categorías: Integralidad en el cuidado en salud; Formación del enfermero en la perspectiva de la integralidad. Conclusión: El cuidado integral es una construcción gradual y se da a partir de relaciones y reformulación de modelos unidimensionales, para ello, es necesario asociar el conocimiento y la práctica para diseminación y aplicabilidad, imprescindible al abordaje desde la formación académica.

Descriptores: Integralidad en salud; Cuidados de enfermería; Enseñanza. 


\section{INTRODUÇÃO}

A integralidade é definida como um dos princípios do Sistema de Saúde Brasileiro, estando inserida como princípio que transcende a prática curativa, compreendendo o ser humano de forma totalitária e não parcial(1).

O conceito, entretanto, vai além de um princípio do SUS, sendo visto como uma ação social, um conjunto articulado e contínuo das ações e serviços preventivos e curativos, sejam eles individuais ou coletivos, de baixa, média ou alta complexidade do sistema ${ }^{(2,3)}$. Para alguns autores, a integralidade é um valor a ser sustentado e defendido nas práticas dos profissionais de saúde, valor este expresso no modo de interação dos profissionais para com os pacientes ${ }^{(4)}$.

A percepção da mudança de visão com relação ao cuidado está inserida nos cursos de graduação de enfermagem, porém, os modelos gerenciais obsoletos, construídos para suprir a demanda de determinada época, ainda são praticados atualmente ${ }^{(5)}$. A inserção da integralidade do cuidado como um dos eixos para uma melhoria na formação do profissional enfermeiro garantiria a qualidade de assistência com um olhar inovador ${ }^{(1)}$.

O perfil de enfermeiros e a sua formação tem influência direta sobre o sistema de saúde, e a implementação de um currículo direcionado para a integralidade do cuidado garantirá aprimorar o atendimento no SUS. A integralidade é o novo paradigma para a formação dos enfermeiros, garantido profissionais que saibam fazer atividades curativas, preventivas, com enfoque clínico, epidemiológico e social, para reinventar o sistema de ensino aprendizagem ${ }^{(3)}$. Tal afirmação já é realidade nas novas grades curriculares, ponto em que se mostra a atualização do sistema de ensino, vistas novas mudanças e necessidades na sociedade.

Os acadêmicos estão sendo preparados para uma melhor formação, tornando-se aptos a fazer a diferença dentro da sociedade. A inserção da integralidade do cuidado como um dos eixos para uma melhoria na formação do profissional enfermeiro é uma garantia da qualidade de assistência com um olhar inovador(1). Lima e colaboradores(b) apontam que a influência da universidade no ensino dos acadêmicos de enfermagem é notória, porém os mesmos necessitam almejar, por si só, uma melhor qualificação e uma transformação no pensamento relacionado à melhoria no atendimento ao indivíduo e à comunidade. A integralidade do cuidado parece estar relacionada ao envolvimento do profissional com o cotidiano do seu trabalho, ao querer fazer melhor e isso vem da sua formação acadêmica.

Desta forma, identifica-se a importância da formação do profissional de enfermagem nos diferentes cenários de cuidado vivenciados pelos estudantes, de forma a propiciarthes uma interação democrática com os sujeitos que cuidam e os que são cuidados, bem como a valorização dos saberes nos diferentes níveis de complexidade do sistema.

Diante disso, delineou-se como questão da pesquisa: Como os acadêmicos de enfermagem percebem a aplicação do princípio da integralidade na sua formação profissional?

A discussão deste tema torna-se relevante, partindo-se do pressuposto que a integralidade do cuidado é o caminho para o alcance de uma melhor qualidade da assistência, bem como está diretamente ligada à formação do sujeito e às experiências vividas na prática acadêmica. Assim, o trabalho poderá contribuir para reflexão do discente sobre o sentido da integralidade do cuidado.

Conhecer a percepção dos acadêmicos de enfermagem sobre integralidade no cuidado, a partir da vivência ao longo do curso, e como ocorre a aplicação desse princípio, constituem-se em objetivos do presente trabalho.

\section{METODOLOGIA}

\section{Tipo de estudo}

Trata-se de um estudo exploratório, de cunho descritivo, com abordagem qualitativa.

\section{Participantes da pesquisa}

Nove acadêmicos de enfermagem recrutados com base nos seguintes critérios de inclusão: maiores de 18 anos, matriculados nas disciplinas do sétimo, oitavo e nono período.

\section{Local do estudo}

A pesquisa foi realizada em uma Universidade do estado do Nordeste.

\section{Coleta dos dados}

A coleta de dados foi realizada no primeiro semestre de 2017, por meio de entrevista individual semiestruturada. 0 roteiro da entrevista foi realizado com base nos objetivos do estudo e nos pressupostos estabelecidos, visando responder ao questionamento da pesquisa. As entrevistas foram agendadas previamente de acordo com a disponibilidade dos envolvidos, e realizadas individualmente pelas pesquisadoras, no campus acadêmico onde o curso é desenvolvido. As entrevistas tiveram um tempo médio de 20 minutos; cada participante foi identificado pelo codinome $A$ de acadêmico seguido de um número, mediante ordem das entrevistas. As entrevistas foram gravadas em mídia digital e, após transcritas, foram organizadas em pastas no computador destinadas a esta finalidade e, posteriormente, analisadas.

\section{Procedimentos de análise dos dados}

A análise dos dados foi realizada com base nas técnicas 
de Minayo7 que contempla três etapas: 1) A primeira etapa compreende a organização do material, onde todas as entrevistas serão transcritas e posteriormente será realizada leitura de todo material em busca de contemplação dos objetivos do estudo; 2) A exploração do material abarca a segunda etapa, na qual, pretende-se obter o núcleo de compreensão do texto; 3) A terceira etapa contempla o tratamento dos resultados e a interpretação, onde serão propostas inferências e interpretações relacionadas com o referencial teórico7.

Partindo da análise dos discursos dos acadêmicos, foram agregadas duas categorias temáticas e duas subcategorias, associando as percepções e vivências dos participantes sobre a integralidade do cuidado (Figura Ol)

Figura 01. Categorização do estudo. Aracaju, 2017.

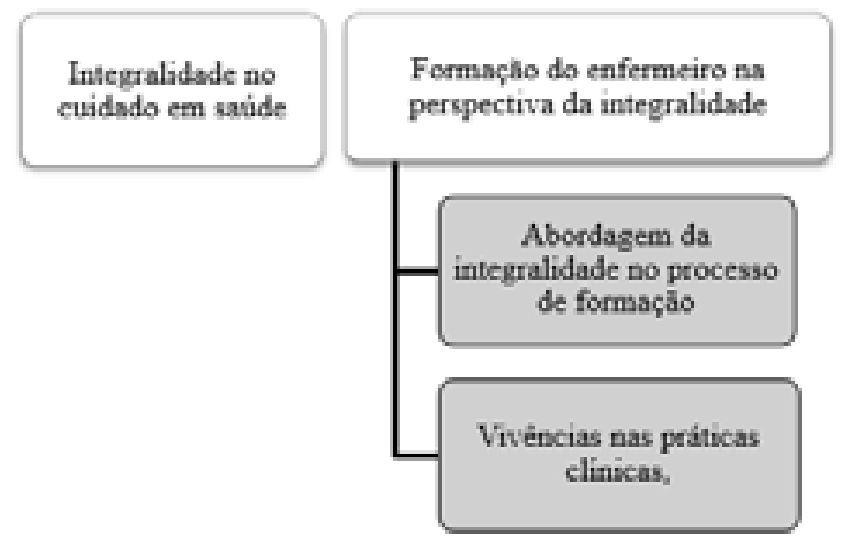

\section{Procedimentos éticos}

O projeto foi submetido ao Comitê de Ética em Pesquisa - Plataforma Brasil e aprovado com parecer de no 1.867.828. Todos os participantes assinaram o Termo de Consentimento Livre e Esclarecido (TCLE).

\section{RESULTADOS}

\section{Integralidade do cuidado em saúde}

A integralidade do cuidado implica em um confronto entre o individual e coletivo; apresenta-se como perspectiva no levantamento das necessidades individuais e coletivas. Essa premissa está presente nos discursos dos acadêmicos, no qual destacam a importância de um atendimento voltado para o físico, mental e espiritual, conforme demonstrado nas seguintes falas: "[...] é ver o indivíduo como um todo, como um ser holístico, vai focar não só na doença, vai focar no psicológico, espiritual, vai focar nisso tudo olhando todas as necessidades" (A3); "[...] prestar uma assistência olhando todos os aspectos que envolvem aquele indivíduo e a sua 'família, tratando dos eixos biopsicossocial daquele indivíduo, e não apenas como se ver muitas vezes, no modelo biomédico em que o cuidado acaba sendo centrado na doença e na cura daquela doença. E deixando de lado outros aspectos que talvez tenha influenciado a melhora desse paciente" (A7).

A integralidade deve atender a população como um todo, observando suas particularidades e prestando um serviço de qualidade: "[...] é uma assistência prestada de forma completa e humanizada ao paciente durante a internação... Oferecendo tudo q ele necessita" (A8); “[...] entender o paciente como um todo não abordando somente a doença, considerado as relações emotivas, saúde mental, meio em que convive espiritual" (A6)

\section{Abordagem da integralidade no processo de formação}

Este eixo refere-se à abordagem e aplicação do principio da integralidade, partindo da matriz curricular e das estratégias de ensino adotadas pelos docentes. Constata-se, entre as falas dos acadêmicos, que a temática em estudo é abordada durante a trajetória do curso, contudo, são nítidas as dificuldades enfrentadas no processo: "Desde o início da graduação somos encaminhados para prestar o cuidado. com as teorias de enfermagem e depois no decorrer do curso aprendemos a aplicá-las" (A9); "Pouco abordada, o que prevalece é a concepção do processo saúde e doença limitando-se em abordar enfermidade e a cura" (A4).

\section{Vivências nas práticas clínicas}

Diante da entrevista, constatou-se a tentativa dos acadêmicos em aplicar o principio da integralidade nas práticas clínicas realizadas durante o curso, quando enfatizam as dificuldades, como é possivel observar nos depoimentos: “[...] infelizmente quando vai para os campos práticos a gente percebe a dificuldade algumas vezes de construir e realizar esse cuidado integral do paciente pelas próprias dificuldades dos campos" (A2); "É perceptível que na rotina profissional é difícil aplicar a integralidade, porém não é impossível. A superlotação dos hospitais, principalmente as públicas, dificulta muito esta assistência integral" (Al).

Pensando no atendimento absoluto, os acadêmicos apontam o diálogo e a humanização como formas de aproximação com a realidade do indivíduo que necessita de cuidado e sinalizam a ausência desses sentimentos no atendimento realizado por muitos profissionais: "[...] aplico a integralidade conversando com o paciente, tratando-o de forma holística, procuro levar em consideração a sua singularidade" (A4); “[...] em todos os ambientes que estagiei senti muita carência, falta empatia, talvez falta de amor pela profissão [...] vi profissionais apenas voltados para técnica, fazendo o cuidado de forma técnica" (A3). 
Nesta perspectiva, o diálogo deve estar presente em todo processo de ensino-aprendizagem de forma horizontal entre os sujeitos, proporcionando aproximação com a realidade, possibilitando o reconhecimento das necessidades dos indivíduos: "[...] É raro de ver a equipe se unir em benefício do paciente. Muitas vezes o cuidado é prestado de forma dissociada, sem comunicação, sem interação interdisciplinar [...]" (A5); “[...] Todo o paciente merece uma assistência humanizada de qualidade, nenhum profissional, estudante é o dono do saber, mas devemos cuidar de forma integral [...] se o nosso objetivo é cuidar do nosso paciente, não tem por que não prestar uma assistência completa" (Al)

As falas dos acadêmicos de enfermagem, quando estimulados a refletir e discutir acerca de estratégias para incentivo do cuidado integral de forma ativa durante a graduação, apontam a necessidade das práticas modulares possibilitando ampliar o olhar quanto às necessidades do paciente, ressaltandotambéma importância dasistematização da assistência para o cuidado efetivo: "Aplicação das práticas em blocos, por exemplo, um mês eu fico em tal unidade todos os dias, assim vou conseguir dar continuidade ao cuidado integral, aplicar a SAE, levantar queixas, diagnósticos de enfermagem planejar os cuidados e avaliar os resultados (Al); "Acredito que uma das melhores formas é através da SAE porque assim a gente consegue analisar o paciente como um todo, traçar os diagnósticos, não só relacionados à doença, mas perceber também a melhora do paciente [...] (A2); "Que as disciplinas sejam em módulo, permitindo maior contato com o paciente maior tempo" [...] (A4)

Considerando a importância de uma assistência de qualidade, pautada no cuidado integral, nos resultados observa-se que as práticas ainda estão muito limitadas ao processo de saúde e doença, deixando em segundo plano a promoção, proteção e recuperação da saúde, bem como um bom relacionamento da equipe e dos usuários.

\section{DISCUSSÃO}

Para atingir um degrau mais elevado da assistência, prezando pelo cuidado integral, os diferentes profissionais detentores dos saberes científico devem compartilhar e traçar seus caminhos, interligando estratégias que visem o benefício dos usuários e uma assistência completa e humanizada ${ }^{(8)}$

A formação profissional não pode ter como referência apenas a doença da pessoa, aplicando de forma única o modelo biomédico que objetiva o cuidado voltado ao diagnóstico e a busca pelo tratamento, mas deve compreender as pessoas de forma ampla, contemplando todas as necessidades apresentadas $^{(9)}$. Chaves e colaboradores afirmam que não se faz mudanças nos modelos assistenciais e gerenciais sem o "querer" dos profissionais de enfermagem(5).
Para que ocorra a aplicação da integralidade é preciso considerá-la como uma extensão das práticas em saúde, a qual resulta de um encontro entre os profissionais e os usuários ${ }^{(1)}$. Os autores Gonzalez e Almeida ${ }^{(10)}$ ressaltam a importância da atuação profissional alinhada ao cuidado total nas práticas de saúde, reconhecendo o contexto social e estruturação dos serviços, podendo estes dificultar ou propiciar a aplicação do agir integral.

Os enfermeiros devem garantir que a prática do cuidado aconteça de forma mais humana possivel; para tal, devem adquirir uma nova postura de trabalho, voltado a um atendimento com maior aproximação, para entender aquele paciente, garantindo um maior vínculo(l)

O uso da integralidade contempla o processo de formação, sendo esse um elemento que necessita ser construído desde o início da formação(1,11). A abordagem e aplicação do princípio da integralidade estão presentes na grade curricular dos cursos de enfermagem ${ }^{(12)}$. Entretanto, são nítidas as dificuldades enfrentadas, como a fragmentação da teoria e prática em algumas disciplinas, não possibilitando meios para o entendimento e aplicação do cuidado integral, a desarticulação do contexto social em que o país está inserido e as necessidades de saúde da população, a necessidade de discussões e reflexões quanto às estratégias de aprendizagem aplicadas e o papel dos envolvidos na formação profissional.

\section{Limitações do estudo}

Os resultados da pesquisa contemplam dados qualitativos, limitando-se a um grupo especifico.

\section{Contribuições do estudo para a prática}

O estudo possibilita uma reflexão no tocante à formação dos enfermeiros. Construir o cuidado integral em saúde sugere adotar práticas como princípio educativo baseado em um novo processo de ensino aprendizagem em saúde, o qual possibilite romper o saber formatado e descontextualizado. Deste modo, é indispensável que as instituições de enfermagem ampliem as discussões e considerem a necessidade de mudanças referente ao perfil do egresso, adequando os currículos e estratégias de ensino.

\section{CONCLUSÃO}

Os resultados desse estudo evidenciam as dificuldades na aplicação da integralidade durante o processo de formação acadêmica, fato que pode influenciar na assistência prestada por esses futuros profissionais. Formar profissionais qualificados a cada dia torna-se um grande desafio, principalmente quando, além de suprir as demandas do mercado, o direcionamento da assistência é voltado para as necessidades dos indivíduos. Outro ponto de destaque na 
pesquisa é a importância das tecnologias leves no contexto da saúde, o que fica evidente quando se observa o saber e o fazer entrelaçados com a escuta e criação de vínculos.

Para se buscar uma assistência baseada na integralidade, é preciso contar com uma equipe capacitada para atender as diversas demandas, articulando com ações de promoção à saúde e prevenção de doenças.

\section{Contribuição dos autores}

Concepção e/ou desenho, análise e interpretação dos dados, redação do artigo, revisão crítica, revisão final: Fernanda Costa Martins Gallotti, Ana Thaís Santana Santos, Caroline Bispo de Oliveira, Aglae da Silva Araujo Andrade, Meiriane do Carmo Passos.

\section{REFERÊNCIAS}

1. Klosh D, Reibnitz KS, Boehs AE, Wosny AM, Lima MM. Principio da integralidade do cuidado: nos projetos politico-pedagógicos dos cursos de Enfermagem; Rev. Latino-Am. Enferm. USP 2014; [cited 2017 Jun 11]; Available from: http://www.scielo.br/pdf/rlae/2014nahead/pt_0104-1169-rlae-0104-1169-3381-2469.pdf

2. Camargo RAA; Zuelli FMGC; Toneti AN, Silva MAI, Gonçalves MFC, Andrade LS. A formação do conceito de integralidade em saúde à luz da abordagem histórico-cultural. Rev. Eletr. Enf. [Internet], [s.I], 2015;17(4)1-10. [cited 2017 Jun 06]; Available from: https://www.fen.ufg.br/revista/v17/n4/pdf/v17n4a08.pdf

3. Carbogim FC, Friedrich DBC, Puschel VAA, Oliveira LB, Nacimento HR. Paradigma da integralidade no curriculo e nas estratégias de ensino em enfermagem: um enfoque histórico-cultural. R. Enferm. Cent. O. Min. 2014;4(1):961-970. [cited 2017 Mai 18]; Available from: http://www.seer.ufsj.edu.br/index.php/recom/ article/view/426

4. Barbosa JAJ.; Perales PGPS.; Vannuchi MTO, Martins EAPP. O princípio da integralidade como norteador da formação do enfermeiro. Espaço para a saúde - Revista de Saúde Pública do Paraná, Londrina, 2016;17(1) 101-106. [cited 2017 Mai 02]; Available from: http://espacoparasaude.fpp.edu.br/index.php/espacosaude/article/viewFile/373/13

5. Chaves LDP, Mininel VA, Silva JAM, Alves LR, Silva MF, Camelo SHH. Supervisão para a Integralidade do cuidados; Rev .Bras, Enferm . 2017; 70(5): 1106-11. [cited 2018 Abr 20]; Available from: http://dx.doi.org/10.1590/0034-7167-2016-0491

6. Lima TC, Paixão FRC, Cândibo EC, Campos CJG, Ceolim MF. Estágio curricular supervisionado: análise da experiência discente; Ver. Bras. Enferm 2014; 67(1): 133-40. [cited 2018 Abr 20]; Available from:

http://www.redalyc.org/html/2670/267030130018.
7. Minayo CSM. Análise qualitativa: teoria, passos e fidedignidade. Ciência \& Saúde Coletiva, 2012, 17(3):621-626.

8. Falkenberg MB, Mendes TPL, Moraes EP, Souza EM. Educação em saúde e educação na saúde: conceitos e implicações para a saúde coletiva. Ciência \& Saúde Coletiva. Brasilia, 2014, 19(3):847852. [cited 2017 Jun 11]; Available from: http://www.scielo.br/pdf/ csc/v19n3/1413-8123-csc-19-03-00847.pdf

9. Silva KL, Sena RR. A formação do enfermeiro: construindo a integralidade do cuidado. Revista Brasileira de Enfermagem 2006; 59(4):488491. [cited 2017 Mai 25]; Available from: http://www.redalyc.org/html/2670/267019620003.

10. Lima MM, Reibnitz KS, Prado ML, Kloh D. Integralidade na formação do Enfermeiro: possibilidades de aproximação com os pensamentos de Freire. Saúde Transform. Soc. Florianópolis, 2013, vol.4 no.4. [cited 2017 Mai 17]; Available from: http://pepsic.bvsalud.org/scielo.php?script=sci_arttext\&pi$d=S 2178-70852013000400003$

11. Franco TB, Merhy EE. El reconocimiento de la producción subjetiva del cuidado salud colectiva 2011; 7(1):9-20. [cited 2017 Jun 01]; Available from: https://www.researchgate.net/publication/287333982_El_reconocimiento_de_la_produccion_subjetiva_del_cuidado

12. Moretti-Pires RO, Bueno SMV. Freire e formação para o sistema único de saúde: o enfermeiro, o médico e o odontólogo. Acta Paul Enferm 2009; 22(4):439-44. [cited 2017 Jun 10]; Available from: http://www2.unifesp.br/acta/pdf/v22/n4/v22n4al5.pdf 